\title{
An analytical model of the influence of cone sensitivity and numerosity on the Rayleigh match
}

\author{
LI ZHAOPING ${ }^{1 *}$ AND JOSEPH CARROLL ${ }^{2}$ \\ ${ }^{1}$ University College London, London, UK \\ ${ }^{2}$ Medical College of Wisconsin, Wisconsin, US \\ *Corresponding author: z.li@ucl.ac.uk \\ Compiled January 13, 2016
}

The Rayleigh match is defined by the range of mixtures of red and green lights that appear the same as an intensity-adjustable monochromatic yellow light. The perceptual match indicates that the red/green mixture and the yellow light have evoked the same respective cone absorptions in the L- and M-cone pathways. Going beyond the existing models (e.g., Pokorny et al 1973, He \& Shevell, 1995; Thomas \& Mollon, 2004; Barbur et al 2008), the Poisson noise in cone absorptions is proposed to make the matching proportion of red-green mixtures span a finite range, since any mixture in that range evokes cone absorptions that do not differ from those by a yellow light by more than the variations in the absorption noise. We derive a mathematical formula linking the match midpoint or match range with the sensitivities and numerosities of the two cones. The noise-free, exact, matching point, close to the mid-point of the matching range, depends only on the L- and M-cone sensitivities to each of the red, green, and yellow lights (these sensitivities in turn depend on the preferred wavelengths $\left(\lambda_{\max }\right)$ and optical densities of the cone pigments and the various pre-receptor retinal light filtering properties). Meanwhile, the matching range depends on both these cone sensitivities and the relative numerosity of the L- and M-cones. The model predicts that, in normal trichromats, all other things being equal, the match range is smallest when the ratio $r$ between $\mathrm{L}$ and $\mathrm{M}$ cone densities is $r=R^{-1 / 2}$ with $R$ as the ratio between the sensitivities of the $L$ and $M$ cones to the yellow light, i.e., when $L$ and $M$ cones are similarly abundant in typical cases, and as $r$ departs from $R^{-1 / 2}$ the match range increases. For example, when one cone type is 10 times more numerous, the match range increases 2-3 fold, depending on the sensitivities of the cones. Testing these model predictions requires either a large data set to identify the effect of one factor (e.g., cone numerosity) while averaging out the effects of the other factors (e.g., cone sensitivities), or for all factors to be known. A corollary of this prediction is that, because they are more likely than usual to have very different $L$ and $M$ cone densities, the matching ranges of normal female trichromats who are carriers of dichromacy (but not anomalous trichromacy) are likely to have a larger matching range than usual, particularly for the deutan carriers. In addition, the model predicts that in strong tetrachromats (whose four dimensions of color are preserved post-receptorally), the Rayleigh matching is either impossible or the matching range is typically smaller than usual. (c) 2016 Optical Society of America

OCIS codes: (330.0330) Vision, color, and visual optics; (330.1690) Color; (330.5310) Vision - photoreceptors

http://dx.doi.org/10.1364/ao.XX.XXXXXX

\section{INTRODUCTION}

The Rayleigh match[1] is a procedure to classify color vision, e.g., to diagnose color-blindness or color vision abnormality. In this procedure, a test subject views a circular field of about $2^{\circ}$ in diameter split into top and bottom halves. The upper hemifield contains a mixture of red and green monochromatic lights (with wavelengths $\lambda_{R} \approx 670 \mathrm{~nm}$ and $\lambda_{G} \approx 545 \mathrm{~nm}$, respectively) of given intensities $R$ and $G$, respectively, and the lower hemifield contains a yellow monochromatic light (of wavelength $\lambda_{Y} \approx 589 \mathrm{~nm}$ ). The red-green mixture is adjusted by having a fraction $f$ of the red light combined with a fraction $(1-f)$ of the green light, and the intensity $Y$ of the yellow light is also adjusted, such that at some values of $f$ and $Y$ the two hemifields are matched in appearance. The range of $f$, and its mid-point, to achieve the match are used to classify color vi- 
sion. Although readouts of typical anomaloscopes, e.g., Nagel's anomaloscope[2], in practice scale the $f$ values to the range between 0 and 70, this paper uses the range $0 \leq f \leq 1$ by the definition of fraction.

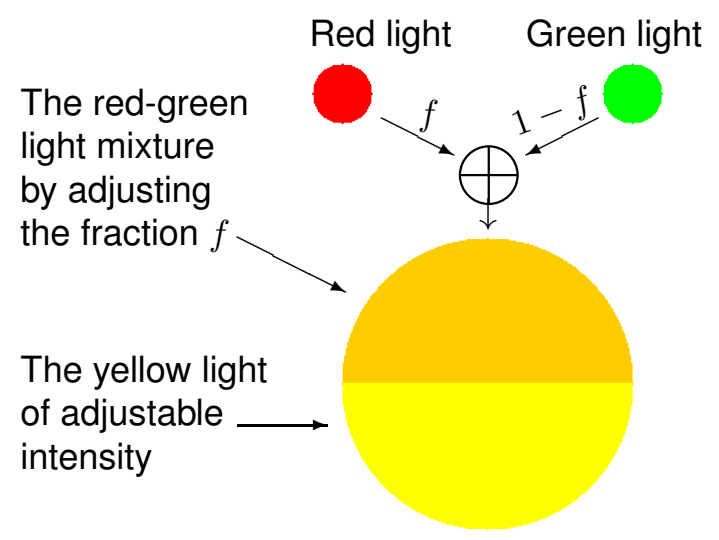

Fig. 1. The Rayleigh match is achieved when the mixture made of a fraction $f$ of the red light and a fraction $1-f$ of the green light appears identical to the yellow light with a suitable intensity.

Normally, humans have three types of cones, sensitive to short (S), medium (M), and long (L) wavelengths of lights, with their sensitivities peaking at the wavelength $\lambda_{\max } \approx 420,530$, and $557 \mathrm{~nm}$, respectively[3]. Hence, the $S$ cones are not appreciably excited by any of the three lights $(R, G, Y)$ in the Rayleigh matching procedure, which thus only classifies color vision characters involving the properties of the $\mathrm{L}$ and $\mathrm{M}$ cones. Normal trichromats, who have normal L and M cones, typically have a small matching range near $f=0.5$ (given a suitable calibration of the light intensities $R$ and $G$ and their wavelengths)[4]. Dichromats lacking L cones (protanopia) or M cones (deuteranopia) can match with any $f$ values. Observers with defective $\mathrm{L}$ (protanomaly) or M (deuteranomaly) cones are called anomalous trichromats. As $\lambda_{\max }$ of the defective L/M cones are shifted towards the $\lambda_{\max }$ of the normal M/L cones, anomalous trichromats typically have a relatively large matching $f$ range with a mid-point $f$ away from the typical $f$ values for the normals.

It has long been recognized that the Rayleigh match is achieved when the two hemifields are identical in terms of their excitations evoked in the normal or abnormal $\mathrm{L}$ and / or M cones of the observer. Accordingly, models of the Rayleigh match have been developed[5-8]. Given the input light from each hemifield, the cone excitations from this hemifield can be calculated from the (wavelength) spectral sensitivity curves of the cones, and these curves in turn depend on the following cone and retinal properties: $\lambda_{\max }$ of the cone pigments, the optical densities of the cone pigments, and the light filtering properties of the optical medium in the retina before input light reaches the cones. These models help to examine how changes in cone and retinal properties, such as $\lambda_{\max }$ and the optical densities of the cone pigments, affect the matching value $f$. In this paper, we present an analytical model aimed to gain additional insights that are not easily achieved using the numerical methods employed by the previous models. Furthermore, we propose that the non-zero matching range of $f$ is caused by the noise in the cone excitations - zero noise would give a zero matching range and an exact matching point $f$ (except in dichromats) and derive a formula for the matching range which has not been modelled previously. In particular, we show that while the exact matching point $f$ depends only on the spectral sensitivities of the cones (given the light properties), the matching range is also influenced by the relative densities of the $\mathrm{L}$ and $\mathrm{M}$ cones on the retina. In addition, we present specific and experimentally testable predictions from this model with some discussions.

\section{AN ANALYTICAL MODEL OF THE RAYLEIGH MATCH}

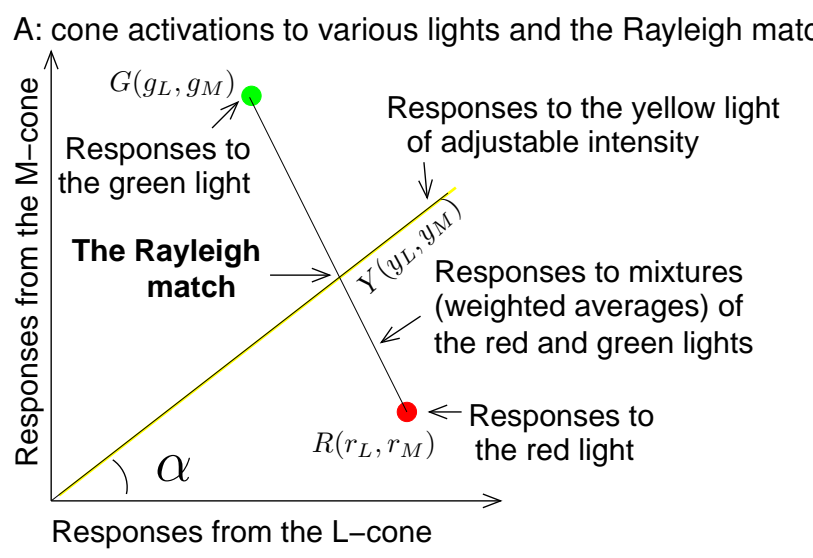

B: components $A$ and $D$ for the cone activations to a mixture

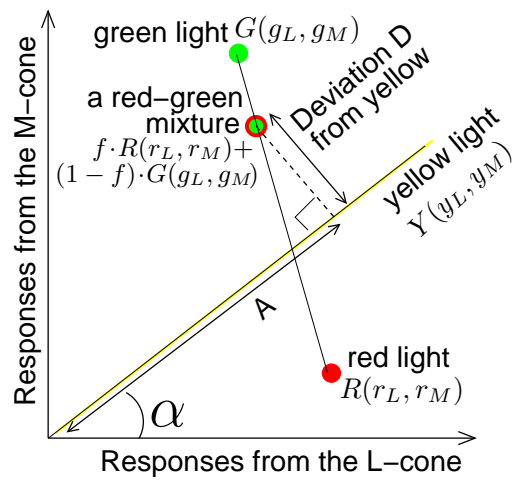

Fig. 2. A: Phase space of $L$ and $M$ cone activations to various lights: red, green, yellow, and red-green mixtures. The Rayleigh match is made when the cone activitations to the yellow light are the same as that to a red-green mixture. The yellow line forms an angle $\alpha$ with the horizontal axis. B: the cone activation to any red-green mixture can be decomposed to two components: A, the component along the yellow light activations, and D, deviation from the yellow light activations. When $\mathrm{D}=0$, the Rayleigh match is achieved. In the text, the responses to the yellow light are $\left(L_{Y}, M_{Y}\right)$; the responses to the red-green mixture light are $\left(L_{R G}, M_{R G}\right)$.

The relevant properties of the retinal cones and the anomaloscope are the following: (1) the pair of fixed intensities $R$ and $G$ for the red and green light respectively, and the adjustable intensity $Y$ of the yellow light, (2) three pairs of sensitivities of the $\mathrm{L}$ and $\mathrm{M}$ cones, respectively, to the three lights: $r_{L}$ and $r_{M}$ to the red light, $g_{L}$ and $g_{M}$ to the green light, and $y_{L}$ and $y_{M}$ to the yellow light, and (3) the pair of fractions, $n_{L}$ and $n_{M}$ (such 
that $n_{L}+n_{M}=1$ ), of the cones that are $\mathrm{L}$ and $\mathrm{M}$ respectively. We will show, analytically and intuitively using graphics, how these five pairs of values (each in a bracket),

$$
(R, G),\left(r_{L}, r_{M}\right),\left(g_{L}, g_{M}\right),\left(y_{L}, y_{M}\right),\left(n_{L}, n_{M}\right),
$$

determine the Rayleigh match and the matching range.

Given the sensitivities $r_{L}$ and $r_{M}$ of the $\mathrm{L}$ and $\mathrm{M}$ cones to the red light, the cone responses to the red light of intensity $R$ is $R r_{L}$ and $R r_{M}$, represented as a point $R\left(r_{L}, r_{M}\right.$ ) (a row vector with components $R r_{L}$ and $R r_{M}$ ) in the two dimensional space of the cone responses in Fig. 2A. Similarly, cone responses to the green light is the point $G\left(g_{L}, g_{M}\right)$ in Fig. 2A. The line connecting these two points contain points representing the cone responses to the red-green mixtures. Let $L_{R G}$ and $M_{R G}$ denote the $\mathrm{L}$ cone and $\mathrm{M}$ cone's responses to a red-green mixture, then, to a mixture with a fraction $0 \leq f \leq 1$ of the red light,

$$
\left(L_{R G}, M_{R G}\right)=f \cdot R\left(r_{L}, r_{M}\right)+(1-f) \cdot G\left(g_{L}, g_{M}\right) .
$$

A $f$ closer to $f=1$ gives a point $\left(L_{R G}, M_{R G}\right)$ closer to $R\left(r_{L}, r_{M}\right)$ on that red-green mixture line in Fig. 2A.

Meanwhile, the cone responses to the yellow lights are

$$
\left(L_{Y}, M_{Y}\right)=Y\left(y_{L}, y_{M}\right),
$$

the points $Y\left(y_{L}, y_{M}\right)$ with various $Y \geq 0$ make another line originating from the origin in Fig. 2A. The Rayleigh matching is defined by

$$
\left(L_{R G}, M_{R G}\right)=\left(L_{Y}, M_{Y}\right),
$$

when the two lines in Fig. 2A intersect each other. Let $\alpha$ be the angle between the yellow line and horizontal, then, a point on this yellow line with a distance $y$ from origin can be written as

$$
\left(L_{Y}, M_{Y}\right) \equiv(y \cos (\alpha), y \sin (\alpha)) .
$$

Fig. 2B shows that any point $\left(L_{R G}, M_{R G}\right)$ on the red-green mixture line can be decomposed to two components, one along the yellow-light line, with a distance $A$ from the origin, and another perpendicular to it with a deviation $D$ to the yellow-light line. Hence, the angle between deviation $D$ and horizontal axis is $\pi / 2+\alpha$, making the unit vector along $D$ as $(-\sin (\alpha), \cos (\alpha))$. Therefore, quantity $D$ is the dot product of vector $\left(L_{R G}, M_{R G}\right)$ with this unit vector,

$$
D=\left(L_{R G}, M_{R G}\right) \cdot(-\sin (\alpha), \cos (\alpha)),
$$

i.e., $D$ is the projection of $\left(L_{R G}, M_{R G}\right)$ on $(-\sin (\alpha), \cos (\alpha))$. The Rayleigh match happens when $D=0$. Using equation (2) and noting

$$
\cos (\alpha)=y_{L} /\left(y_{L}^{2}+y_{M}^{2}\right)^{1 / 2}, \quad \sin (\alpha)=y_{M} /\left(y_{L}^{2}+y_{M}^{2}\right)^{1 / 2},
$$

we have

$$
\begin{aligned}
D & =\frac{1}{\left(y_{L}^{2}+y_{M}^{2}\right)^{1 / 2}}\left[Q_{2}-f\left(Q_{1}+Q_{2}\right)\right], \text { in which } \\
Q_{1} & \equiv R\left(r_{L} y_{M}-r_{M} y_{L}\right), \quad Q_{2} \equiv G\left(g_{M} y_{L}-g_{L} y_{M}\right)
\end{aligned}
$$

and analogously $Q_{3}, Q_{4}, Q_{5}$, and $Q_{6}$, introduced later, are quantities built from our five pairs of values in equation (1). The Rayleigh matching is to adjust the fraction $f$ to make $D=0$ and adjust the yellow light intensity to make $y=A$. Solving $D=0$ for the matching $f$ value gives

$$
f_{\text {match }}=\frac{1}{1+\left(Q_{1} / Q_{2}\right)} .
$$

Therefore, given the red and green light intensities $R$ and $G$, and the three pairs of $\mathrm{L}$ and $\mathrm{M}$ cone sensitivities $\left(r_{L}, r_{M}\right),\left(g_{L}, g_{M}\right)$, and $\left(y_{L}, y_{M}\right)$ to the three lights, the matching fraction $f_{\text {match }}$ is determined by the above formula.

The three pairs of cone sensitivities can be read out from the spectrum sensitivity curves $S_{L}(\lambda)$ and $S_{M}(\lambda)$, respectively, of the $L$ and $M$ cones, as the sensitivity values $S_{L, M}(\lambda)$ for the wavelengths $\lambda=\lambda_{R}, \lambda_{G}$, and $\lambda_{Y}$ of the three monochromatic light sources, see Fig. 3. (For general, non-monochromatic lights, the three pairs of sensitivities is generally, e.g., $r_{L}=$ $\int d \lambda S_{L}(\lambda) I_{i}(\lambda)$ for the input spectrum $I_{i}(\lambda)$, with $\int d \lambda I_{i}(\lambda)=$ 1 , of the light source $i=R, G, Y)$. The sensitivity spectrum $S_{L, M}(\lambda)$ in turn depend on the $\lambda_{\max }$ and optic density $O_{L, M}$ of the photopigment for the cone, and the optical density spectra $O_{\text {lens }}(\lambda)$ of the retina lens and $O_{\text {macular }}(\lambda)$ of the macular layer as follows. For cone $C=L$ or $M$,

$$
\begin{aligned}
& S_{C}(\lambda)=\left(1-10^{-O_{C} \cdot E\left(\lambda, \lambda_{\text {max }}\right)}\right) 10^{-O_{\text {lens }}(\lambda)-O_{\text {macular }}(\lambda),} \\
& \text { with } \quad E\left(\lambda, \lambda_{\text {max }}\right)=\left(e^{E_{1}}+e^{E_{2}}+e^{E_{3}}+0.655\right)^{-1}, \\
& E_{1}=70\left(0.88-\frac{\lambda_{\text {max }}}{\lambda}\right), \\
& E_{2}=28.5\left(0.924-\frac{\lambda_{\text {max }}}{\lambda}\right), \\
& E_{3}=-14.1\left(1.104-\frac{\lambda_{\text {max }}}{\lambda},\right)
\end{aligned}
$$

where $O_{C}$ is the optical density of the photopigment and $E\left(\lambda, \lambda_{\max }\right)$ is called the extinction coefficient of the cone[9]. In typical human eyes, $O_{\text {lens }}(\lambda)$ and $O_{\text {macular }}(\lambda)$ are negligible at the wavelengths of our concern. Hence a cone's sensitivity curve is largely determined by its $\lambda_{\max }$ and optical density $O_{C}$ of the pigment. As summarized in Fig 4, variations of $\lambda_{\max }$ (caused by genes, even among normal trichromats) and $O_{C}$ lead to variations in the three pairs of cone sensitivities $\left(r_{L}, r_{M}\right)$, $\left(g_{L}, g_{M}\right)$, and $\left(y_{L}, y_{M}\right)$, which in turn (by equation (10)) cause variations in the Rayleigh match $f_{\text {match }}$ between different observers.

Let us define our standard trichromat as one with the following $\lambda_{\max }$ and optical density $\mathrm{O}_{\mathrm{C}}$ (slightly different from the example in Fig. 3) of the pigment for the cones and the prereceptor properties:

$$
\begin{aligned}
& \text { L cone } \lambda_{\text {max }}=557(\mathrm{~nm}), \quad O_{L}=0.4, \\
& M \text { cone } \lambda_{\text {max }}=530(\mathrm{~nm}), \quad O_{M}=0.4,
\end{aligned}
$$

Pre-receptor: $O_{\text {lens }}(\lambda)$ and $O_{\text {macular }}(\lambda)$ are as in[10].

Let us also define our standard anomaloscope as the one with

The three primaries with $\lambda_{R}, \lambda_{G}, \lambda_{Y}=670,545,589 \mathrm{~nm}$,

The light intensities $R$ and $G$ give $R / G \approx 23.4$ and $G=1$.

We note from equations (9) and (10) that $f_{\text {match }}$ depends only on the ratio $R / G$ and not $R$ and $G$ individually. Hence, given $R / G$, we can arbitrarily set $G=1$, as we did above, without affecting $f_{\text {match }}$ (although the yellow-light intensity needed for the matching scales with $G$ ). This $R / G$ ratio above is for the "deutan mode" of the anomalosope calibration[6] so that the red and green light excites the $\mathrm{L}$ cone equally (i.e., $R r_{L}=G g_{L}$ ) for our standard trichromat defined above. Our standard trichromat should thus obtain $f_{\text {match }} \approx 0.5$ using our standard anomaloscope. 


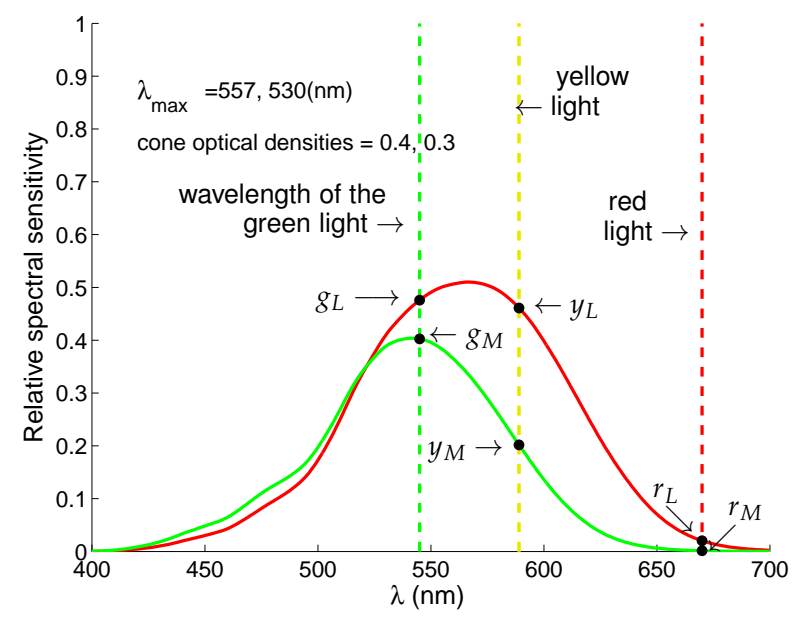

Fig. 3. The spectral sensitivities $S_{L}(\lambda)$ and $S_{M}(\lambda)$ of the $L$ and $M$ cones by equation (11), and illustrations of of the three pairs of sensitivities $\left(r_{L}, r_{M}\right),\left(g_{L}, g_{M}\right)$, and $\left(y_{L}, y_{M}\right)$ to the three lights. Different $\lambda_{\max }$ and optical densities of the cones can alter these six numbers. These six numbers (and the intensities $R$ and $G$ of the red and green light) determine the matching point $f_{\text {match }}$, but the matching range depends in addition on the $\mathrm{L} / \mathrm{M}$ cone density ratio.

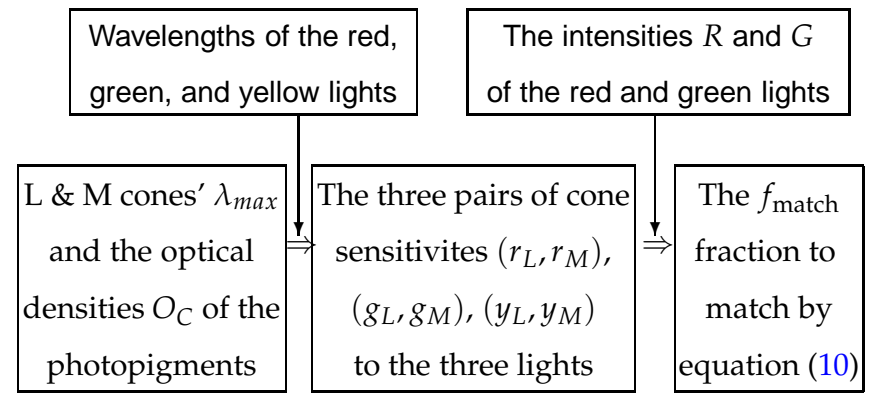

Fig. 4. Summary of how retinal and cone properties determine the outcome of the Rayleigh match.

\section{A. Any fraction $f$ achieves a match for dichromats}

One way to view dichromats lacking the $\mathrm{L}$ or $\mathrm{M}$ cone is to consider their $\mathrm{L}$ and $\mathrm{M}$ cones as identical to each other. For example, consider protanopia as when the L cones are just copies of the $\mathrm{M}$ cones. Hence, these dichromats have $r_{L}=r_{M}, g_{L}=g_{M}$, $y_{L}=y_{M}$, and, from equation (7), $\cos (\alpha)=\sin (\alpha)$. Hence, $Q_{1}=Q_{2}=0$ by equation ((9), and consequently any fraction $f$ gives $D=0$ by equation (8) to achieve the match. Graphically, $r_{L}=r_{M}, g_{L}=g_{M}$, and $y_{L}=y_{M}$ together means that, in Fig. $2 \mathrm{~A}$, the line for the red-green mixtures and that for the yellow lights are both on the diagonal line from the origin $\left(45^{\circ}\right.$ from horizontal), so that all the red-green mixture points are on the line for the yellow lights.

\section{B. Protanomaly requires more red light to match}

The light intensities $R$ and $G$ are calibrated such that quantities in equation (10) for normal trichromats give $Q_{1} \sim Q_{2}$, making $f_{\text {match }}=1 /\left(1+Q_{1} / Q_{2}\right) \sim 0.5$. In protanomaly, the $\lambda_{\text {max }}$ of the $\mathrm{L}$ cones is abnormally reduced towards the $\lambda_{\max }$ of the (normal) $\mathrm{M}$ cones, altering $r_{L}, g_{L}$, and $y_{L}$ accordingly. Because (see Fig. 3) the $r_{L}$ value is very near the tail of L cone's spectral sensitivity curve while $g_{L}$ and $y_{L}$ are near the peak of this curve, a reduction in $\lambda_{\max }$ for the L cones reduces $r_{L}$ by a large percentage while leaving $g_{L}$ and $y_{L}$ relatively unchanged in percentage. Consequently, $Q_{1}=R\left(r_{L} y_{M}-r_{M} y_{L}\right)$ is much reduced while $Q_{2}=G\left(g_{M} y_{L}-g_{L} y_{M}\right)$ is relatively unchanged, making $f_{\text {match }}$ larger to require more red light for the match as observed in observers with protanomaly. Fig. 6A \& B illustrate how a protanomalous differs from normal in the match.

\section{Deuteranomaly requires more green light to match}

In deuteranomaly, the $\lambda_{\max }$ of the $\mathrm{M}$ cones is abnormally increased towards the $\lambda_{\max }$ of the (normal) L cones. Among the affected sensitivities $r_{M}, g_{M}$, and $y_{M}$, only the $g_{M}$ value sits near the peak of the cone sensitivity spectrum. As a result, percentage-wise, $r_{M}$ and $y_{M}$ are both much increased while $g_{M}$ is relatively unchanged. Consequently, $Q_{2}=G\left(g_{M} y_{L}-g_{L} y_{M}\right)$ is much reduced whereas $Q_{1}=R\left(r_{L} y_{M}-r_{M} y_{L}\right)$ is less likely to change by as much in percentage, leading to a reduced $f_{\text {match }}$ to require more green light for the match as observed in observers with deuteranomaly. Fig. 6A \& C illustrate how a deuteranomalous differs from normal in the match.

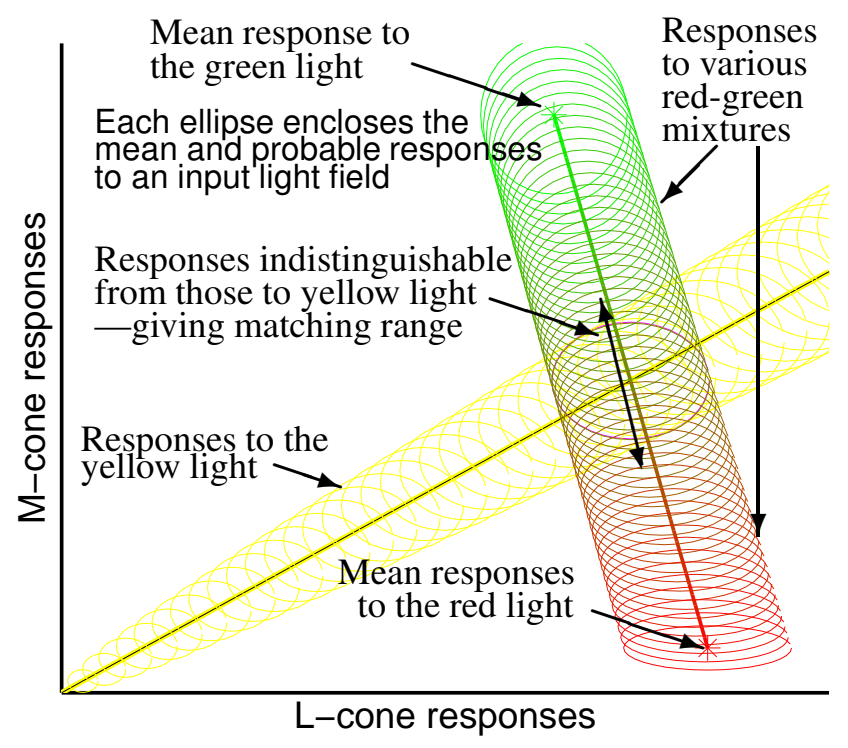

Fig. 5. Illustration of how noise in cone responses lead to a finite, non-zero, range of matching fractions. Each ellipse schematically illustrates the range of probable cone responses that could be evoked by an input light whose average evoked cone responses is marked by the center of the ellipse. The yellow ellipses enclose probable responses to the yellow light; red, green, and red/green ellipses enclose probable responses to the red, green, and mixture lights. The horizontal and vertical extents, respectively, of each ellipse visualize the standard deviations of the noises in $\mathrm{L}$ and $\mathrm{M}$ cones. When two input light fields give substantially overlapping response ellipses, these two inputs match perceptually, i.e., not perceptually distinguishable. Hence, a finite range of red-green mixtures can match the yellow light fields. 


\section{Matching range from cone absorption noise}

Cone absorptions are noisy. Each input, whether a red-green mixture or a yellow light, can evoke many possible pairs of $\mathrm{L}$ and $\mathrm{M}$ cone responses randomly distributed around the mean response pair. Consequently (see Fig. 5), responses to the mixtures and yellow lights are no longer confined to, but distributed around, the two lines of zero-thickness in the twodimensional L-M response space. This is as if the lines become thicker by the typical magnitudes of the cone response noise. The noise blurs small differences between two input fields so that a red-green mixture could appear identical to a yellow light even if the mixing fraction $f$ deviates a little from $f_{\text {match }}$. Hence, a range of $f$ values around $f_{\text {match }}$ can produce mixtures to match the yellow light.

Consider a red-green mixture with a $f=f_{\text {match }}+\Delta f$. Let the zero-noise L-M cone responses to this mixture deviates by a displacement $D$ from the zero-noise responses to the yellow light (see Fig. 2B). Cone noise makes $D$ also a noisy variable, with an average $\bar{D}$ given by equation (8) and a standard deviation $\sigma_{D}$ determined by the noises in both the $\mathrm{L}$ and $\mathrm{M}$ responses. When $|\bar{D}| \leq \sigma_{D}$, i.e., the average $D$ is within the range of the noise blurs, the red-green mixture should appear indistinguishable from the nearest yellow light.

Using equation (8) and noting that $\bar{D}=0$ for $f=f_{\text {match }}$ by definition, we have $\bar{D} \propto \Delta f$ as

$$
\bar{D}=\frac{1}{\left(y_{L}^{2}+y_{m}^{2}\right)^{1 / 2}}(\Delta f)\left(Q_{1}+Q_{2}\right)
$$

The largest two $|\Delta f|^{\prime}$ s, one for a negative $\Delta f_{1}$ and one for a positive $\Delta f_{2}$, to make $\bar{D}^{2}=\sigma_{D}^{2}$ should make the matching range in $f \in\left(f_{\text {match }}+\Delta f_{1}, f_{\text {match }}+\Delta f_{2}\right)$, making the matching range

$$
f_{\text {matching-range }} \equiv \Delta f_{2}-\Delta f_{1} \text {. }
$$

Noise in cone absorption is Poisson like[11], so that the variances $\sigma^{2}\left(L_{R G}\right)$ and $\sigma^{2}\left(M_{R G}\right)$ of noise in $\mathrm{L}$ and $\mathrm{M}$ cone responses, respectively, scale with the respective average responses, $L_{R G}$ and $M_{R G}$,

$$
\sigma^{2}\left(L_{R G}\right)=L_{R G}, \quad \sigma^{2}\left(M_{R G}\right)=M_{R G},
$$

(more accurately, noise variance scales with average response by a Fano factor larger than unity[11], but this discrepancy will be absorbed by our parameter $\beta$ introduced later). In addition, noise in the $\mathrm{L}$ and $\mathrm{M}$ cones are independent of each other. Therefore, since $D$ is a weighted sum of $L$ and $M$ cone responses (with weights $-\sin (\alpha)$ and $\cos (\alpha)$, see equation (8)), the variance $\sigma_{D}^{2}$ in $D$ is also a weighted sum (with weights $\sin ^{2}(\alpha)$ and $\cos ^{2}(\alpha)$ ) of the respective variances in the $L$ and $M$ responses:

$$
\begin{aligned}
\sigma_{D}^{2} & =\sigma^{2}\left(L_{R G}\right) \sin ^{2}(\alpha)+\sigma^{2}\left(M_{R G}\right) \cos ^{2}(\alpha) \\
& =L_{R G} \sin ^{2}(\alpha)+M_{R G} \cos ^{2}(\alpha) .
\end{aligned}
$$

Using equations (2) and (7), and $f=f_{\text {match }}+\Delta f$, we have

$$
\begin{aligned}
\sigma_{D}^{2} & =\frac{1}{y_{L}^{2}+y_{m}^{2}}\left\{\left(\Delta f+f_{\text {match }}\right)\left(Q_{3}-Q_{4}\right)+Q_{4}\right\}, \text { in which } \\
Q_{3} & \equiv R\left(r_{L} y_{M}^{2}+r_{M} y_{L}^{2}\right), \quad Q_{4} \equiv G\left(g_{L} y_{M}^{2}+g_{M} y_{L}^{2}\right)
\end{aligned}
$$

From equation (14), $|\bar{D}|^{2}=\sigma_{D}^{2}$ gives (noting that $f_{\text {match }}=$ $\left.1 /\left(1+Q_{1} / Q_{2}\right)\right)$

$$
(\Delta f)^{2}\left(Q_{1}+Q_{2}\right)^{2}+\left(Q_{4}-Q_{3}\right) \Delta f-\frac{Q_{2} Q_{3}+Q_{1} Q_{4}}{Q_{1}+Q_{2}}=0 .
$$

Since the quantities $Q_{1}, Q_{2}, Q_{3}$, and $Q_{4}$ are built from four pairs of values, $(R, G),\left(r_{L}, r_{M}\right),\left(g_{L}, g_{M}\right)$, and $\left(y_{L}, y_{M}\right)$, the two solutions $\Delta f_{1}$ and $\Delta f_{2}$ to give the matching range (by equation (15)), are determined by these four pairs of values.

So far, we have ignored the fact that many $\mathrm{L}$ and $\mathrm{M}$ cones on the retina receive the visual input from the light fields. While the number of cones does not matter for $f_{\text {match }}$ it does for $\Delta f$ because pooling responses from many cones can smooth out the noise. Let there be $N_{L}$ L cones and $N_{M} \mathrm{M}$ cones responding to each light field, define $N \equiv N_{L}+N_{M}$ and $n_{L} \equiv N_{L} / N$ and $n_{M} \equiv N_{M} / N$. Pooling the cones means to multiply sensitivities $\left(r_{L}, g_{L}, y_{L}\right)$ by $N n_{L}$ and $\left(r_{M}, g_{M}, y_{M}\right)$ by $N n_{M}$ in calculating $\bar{D}^{2}$ and $\sigma_{D}^{2}$. In addition, define $\hat{Q}_{i}$ for each $i=1,2,3,4$ as the $Q_{i}$ when each $r_{L}, g_{L}$, or $y_{L}$ is replaced by $n_{L} r_{L}, n_{L} g_{L}$, or $n_{L} y_{L}$, respectively, and each $r_{M}, g_{M}$, or $y_{M}$ is replaced by $n_{M} r_{M}, n_{M} g_{M}$, or $n_{M} y_{M}$, respectively. Then equation (20) becomes

$$
N(\Delta f)^{2}\left(\hat{Q}_{1}+\hat{Q}_{2}\right)^{2}+\left(\hat{Q}_{4}-\hat{Q}_{3}\right) \Delta f-\frac{\hat{Q}_{2} \hat{Q}_{3}+\hat{Q}_{1} \hat{Q}_{4}}{\hat{Q}_{1}+\hat{Q}_{2}}=0 .
$$

Then, an $N \rightarrow \infty$ requires $\Delta f \rightarrow 0$, since the signal-to-noise diverges when responses are pooled over infinitely many cones. However, our central brain does not seem to be able to integrate signals over an infinitely many cones or over an infinitely long viewing duration to smooth out noise[12-14], otherwise, all signal-to-noise in the central decision stage will approach infinity to give infinite accuracy to color matching and other similar sensory matching tasks. This limit in our central brain is implemented here by replacing an $N \rightarrow \infty$ by a non-infinite parameter $\beta$. Hence, replacing $N$ by $\beta$ in the above equation and solving for $\Delta f$ from it,

$\Delta f=\frac{\hat{Q}_{3}-\hat{Q}_{4} \pm \sqrt{\left(\hat{Q}_{3}-\hat{Q}_{4}\right)^{2}+4 \beta\left(\hat{Q}_{1}+\hat{Q}_{2}\right)\left(\hat{Q}_{2} \hat{Q}_{3}+\hat{Q}_{1} \hat{Q}_{4}\right)}}{2 \beta\left(\hat{Q}_{1}+\hat{Q}_{2}\right)^{2}}$.

Hence, by equation (15),

$f_{\text {matching-range }}=\frac{\sqrt{\left(\hat{Q}_{3}-\hat{Q}_{4}\right)^{2}+4 \beta\left(\hat{Q}_{1}+\hat{Q}_{2}\right)\left(\hat{Q}_{2} \hat{Q}_{3}+\hat{Q}_{1} \hat{Q}_{4}\right)}}{\beta\left(\hat{Q}_{1}+\hat{Q}_{2}\right)^{2}}$,

which can be approximated for normal trichromats (because their $\left(\hat{Q}_{3}-\hat{Q}_{4}\right)^{2}$ is relatively negligible) by

$$
\begin{aligned}
f_{\text {matching-range }} & \approx 2\left(\frac{\hat{Q}_{2} \hat{Q}_{3}+\hat{Q}_{1} \hat{Q}_{4}}{\beta\left(\hat{Q}_{1}+\hat{Q}_{2}\right)^{3}}\right)^{1 / 2} \\
& =\frac{2}{\sqrt{\beta}}\left(\frac{Q_{5}}{\left(Q_{1}+Q_{2}\right)^{3}}\right)^{1 / 2}\left(\frac{y_{L}}{n_{M}}+\frac{y_{M}}{n_{L}}\right)^{1 / 2}
\end{aligned}
$$$$
\text { in which } \quad Q_{5} \equiv G R y_{L} y_{M}\left(r_{L} g_{M}-r_{M} g_{L}\right) \text {. }
$$

Let us extend the definition of our standard trichromat in equation (12) to have relative $\mathrm{L}$ and $\mathrm{M}$ cone densities such that $n_{L}=2 / 3$ (so that $n_{M}=1 / 3$ ), and let this trichromat to have $f_{\text {matching-range }}=0.04$ using our standard anomaloscope in equation (13). Then $\beta \approx 13135$ is required for this $f_{\text {matching-range- }}$ We can use this numerical $\beta$ value in equation (25) or (23) for $f_{\text {matching-range }}$ of general observers with normal or abnormal color vision, if we assume that this central limiting parameter $\beta$ is the same across different observers.

\section{E. Variable matching ranges for anomalous trichromats}

By equation (23), the matching range $f_{\text {matching-range increases }}$ when the denominator $\beta\left(\hat{Q}_{1}+\hat{Q}_{2}\right)^{2}$ in the right-hand side of 
this equation decreases. Because $\hat{Q}_{1} \propto r_{L} y_{M}-r_{M} y_{L}$ and $\hat{Q}_{2} \propto$ $g_{M} y_{L}-g_{L} y_{M}$ (see equation (9)), we have $\hat{Q}_{1} \approx 0$ and $\hat{Q}_{2} \approx 0$ when $r_{L} \approx r_{M}, g_{L} \approx g_{M}$, and $y_{L} \approx y_{M}$, i.e., when the L and $\mathrm{M}$ cones are very similar. Hence, $\hat{Q}_{1}+\hat{Q}_{2}$, and thus the denominator, approaches zero in anomalous trichromats, whose $\mathrm{L}$ and $\mathrm{M}$ cones have very similar $\lambda_{\max }$ (assuming that the two cone types have the same optical density). Therefore, these observers are likely to have larger matching ranges than normal.

This qualitative conclusion still holds when the $\mathrm{L}$ and $\mathrm{M}$ cones have different optical densities, $O_{L}$ and $O_{M}$, for the photopigments. This is because, approximately, the effect of optical densities (see equation (11)) merely scale the $L$ cone sensitivities $r_{L}, g_{L}$, and $y_{L}$ by one factor associated with $O_{L}$ and scale the $M$ sensitivities $r_{M}, g_{M}, y_{M}$ by another factor associated with $O_{M}$. Hence, even when $r_{L} \approx r_{M}, g_{M} \approx g_{L}$, and $y_{L} \approx y_{M}$ no longer hold, $r_{L} y_{M} \approx r_{M} y_{L}$ and $g_{M} y_{L} \approx g_{L} y_{M}$ still hold to make $\hat{Q}_{1} \approx 0$ and $\hat{Q}_{2} \approx 0$.

Fig. 6 shows a graphical understanding of the above conclusion. For protanomalous or deuteranomalous, the ratio L cone response : $\mathrm{M}$ cone response is nearly a constant across input variations, because $\mathrm{L}$ and $\mathrm{M}$ cones have very similar $\lambda_{\max }$ (this constant is one when $\mathrm{L}$ and $\mathrm{M}$ pigments have the same optical density). For protanomalous (Fig. 6B), this makes the line for red-green mixtures and the line for the yellow lights nearly parallel or identical to each other, making more mixtures indistinguishable from the yellow lights. For deuteranomalous (Fig. $6 \mathrm{C})$, this makes the cone responses nearly invariant to the fraction changes in the red-green mixture. In particular, when the anomaloscope is calibrated at or around the deutan mode, defined as when the red and green lights excite a standard L cone equally, the similarity in spectral sensitivity of the two cone mechanisms in deuteranomalous individuals ( $\mathrm{L}$ and $\mathrm{L}^{\prime}$ ) means that the response of neither the $\mathrm{L}$ nor the $\mathrm{L}^{\prime}$ cone varies substantially with the fraction changes in the red-green mixture. Hence, more compositions of the red-green mixture can appear identical to the matched yellow lights when noise blurs small response differences.

Large matching ranges in anomalous trichromats have been observed experimentally[15]. However the matching behavior of color deficient observers is highly variable so that some have small matching ranges[16-18]. This could be understood by noting that a smaller denominator $\beta\left(\hat{Q}_{1}+\hat{Q}_{2}\right)^{2}$ in the formula for $f_{\text {matching-range }}$ in equation (23) makes the value of

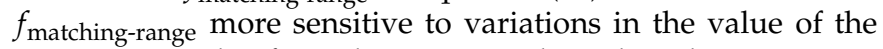
numerator in this formula. In particular, when the numerator is too small, a small $f_{\text {matching-range }}$ results, even though in general a smaller denominator yields a larger $f_{\text {matching-range. Pin- }}$ pointing $f_{\text {matching-range }}$ for an individual requires knowing both the sensitivities and numerosities of the $\mathrm{L} / \mathrm{M}$ cones present in a given retina. In any case, because the denominator is smaller for anomalous trichromats than that for the normals, $f_{\text {matching-range }}$ is not only more likely larger, but also more highly variable, among anomalous trichromats.

\section{F. Match range increases for normal trichromats when the numbers of $L$ and $M$ cones differ by too much}

Because $f_{\text {matching-range }}$ also depends on the relative cone densities $n_{L}$ and $n_{M}$ (see equation (25) or (23)), normal trichromats can also have larger $f_{\text {matching-range }}$ for some $n_{L} / n_{M}$. To study the effect of $n_{L} / n_{M}$ in normal trichromat, we use the approxi-

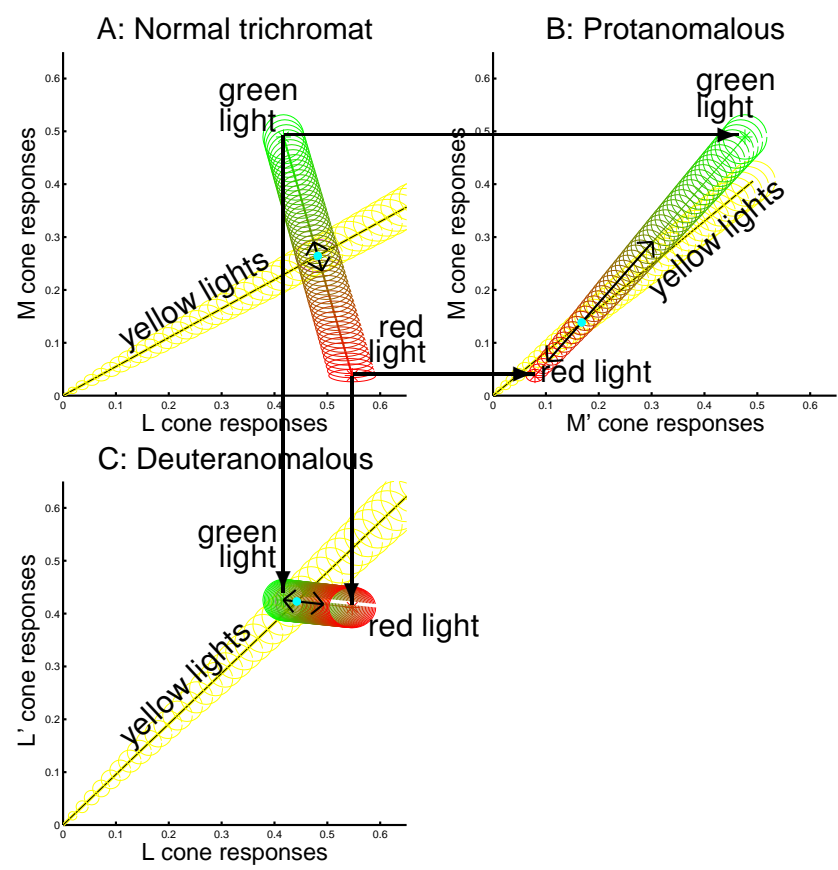

Fig. 6. Comparing matching in a normal trichromat (A), a protanomalous (B), and a deuteranomalous (C). The two horizontal black arrows from A panel to B panel indicate that the $\mathrm{M}$ cone responses in protanomalous are copies of those in normal trichromats. Similarly, the two vertical black arrows from A panel to $C$ panel indicate that the $\mathrm{L}$ cone responses in deuteranomalous are copies of those in normal trichromats. The abnormal cone is the $\mathrm{M}^{\prime}$ cone in panel $\mathrm{B}$ and $\mathrm{L}^{\prime}$ cone in panel C. In protanomalous (B) and deuteranomalous (C), the two cone types have very similar responses for any typical input (when the two cone pigments have the same optical density). For protanomalous (B), this makes the line for the yellow light and the line for the red-green mixtures nearly parallel or identical to each other. For deuteranomalous (C), this makes cone responses insensitive to changes in the fraction $f$ of the redgreen mixture (because intensities of red and green lights in typical anomaloscope are calibrated to evoke similar L cone responses). In each panel, the cyan dot marks the matching point, and the black double arrow spans the matching range schematically; the length of the black arrow as a fraction of the total distance between the mean responses to the red and green lights is the numerical matching range (e.g., in both $B$ and $C, f_{\text {matching-range }} \sim 0.5$ ).

mation of $f_{\text {matching-range }}$ by equation (25), re-written here:

$$
f_{\text {matching-range }} \approx \frac{2}{\sqrt{\beta}}\left(\frac{Q_{5}}{\left(Q_{1}+Q_{2}\right)^{3}}\right)^{1 / 2}\left(\frac{y_{L}}{n_{M}}+\frac{y_{M}}{n_{L}}\right)^{1 / 2},
$$

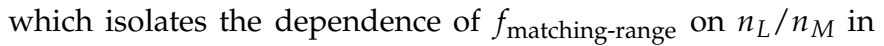
the factor (note that $n_{L}+n_{M}=1$ )

$$
Q_{6} \equiv\left(\frac{y_{L}}{n_{M}}+\frac{y_{M}}{n_{L}}\right)^{1 / 2}=\left(\frac{y_{L}}{1-n_{L}}+\frac{y_{M}}{n_{L}}\right)^{1 / 2},
$$

the other $(\ldots)^{1 / 2}$ factor in $f_{\text {matching-range }}$ depends only on the light intensities ( $R$ and $G$ ) and the three pairs of cone sensitivi- 
A: the Rayleigh matching with four different $n_{L} / n_{M}$ 's

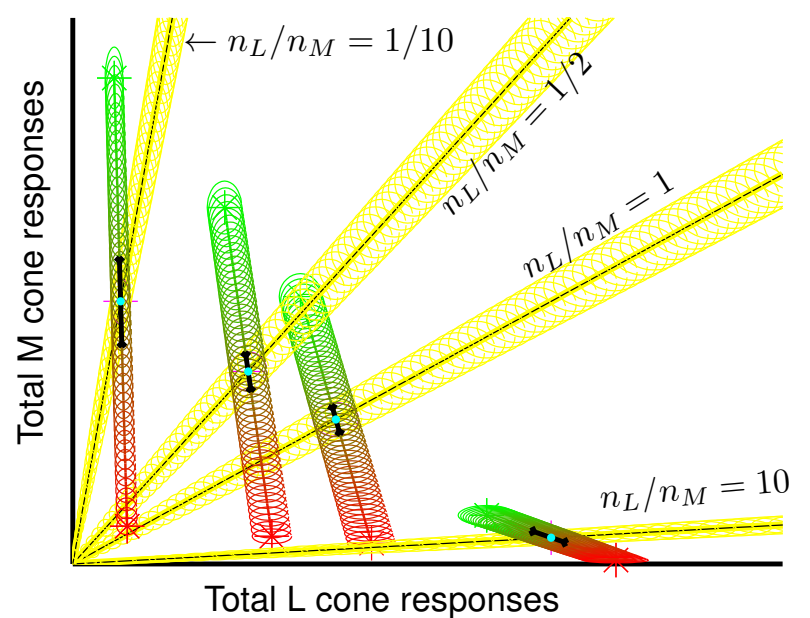

B: Scale of $f_{\text {matching-range versus }} n_{L}$

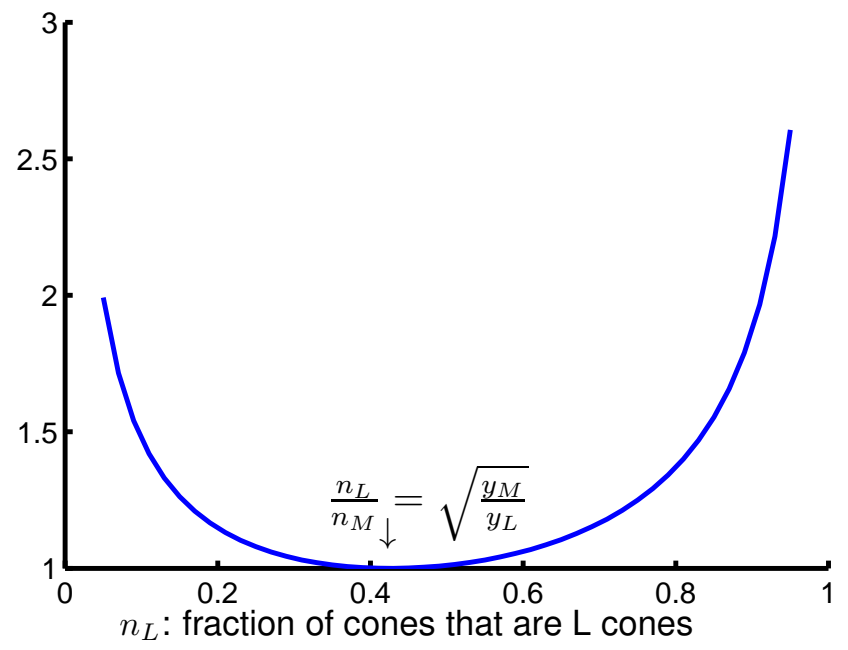

Fig. 7. Effects of the relative $L$ versus $M$ cone numerosities on the matching range $f_{\text {matching-range }}$ A: schematic illustrations of four examples of normal trichromats with the same three pairs of sensitivities $\left(r_{L}, r_{M}\right),\left(g_{L}, g_{M}\right)$, and $\left(y_{L}, y_{M}\right)$, but different relative $\mathrm{L}$ and $\mathrm{M}$ cone numerosities. The black double arrows schematically spans the matching range, the cyan dots mark the $f_{\text {match }}$ locations. B: the $f_{\text {matching-range }}$ relative to its minimum value (which occurs at $n_{L}=\frac{\sqrt{y_{M}}}{\sqrt{y_{L}}+\sqrt{y_{M}}}$ ) versus $n_{L}(=0.05-0.95)$, showing that, all other things being equal, $f_{\text {matching-range }}$ increases when $\mathrm{L}$ cones are too many or two few relatively. $y_{L} / y_{M}=1.82$ in both $\mathrm{A}$ and $\mathrm{B}$.

ties $\left.\left(r_{L}, r_{M}\right),\left(g_{L}, g_{M}\right),\left(y_{L}, y_{M}\right)\right)$. Solving $d Q_{6} / d n_{L}=0$ gives:

with all other things being equal,

$f_{\text {matching-range }}$ is minimum when $n_{L}=\frac{\sqrt{y_{M}}}{\sqrt{y_{L}}+\sqrt{y_{M}}}$,

see Fig. 7. A 10 fold change in $n_{L} / n_{M}$ from a typical $n_{L} / n_{M} \approx$ 1 can increase $f_{\text {matching-range }}$ by two or more fold, more so when $y_{L} / y_{M}$ deviates more from unity. Fig. 7A illustrates the Rayleigh matching in four example values of $n_{L} / n_{M}$ when
$\left(r_{L}, r_{M}\right),\left(g_{L}, g_{M}\right),\left(y_{L}, y_{M}\right)$ are fixed. Intuitively, because the ratio (total $\mathrm{L}$ cone response) : (total $\mathrm{M}$ cone response) scales with $n_{L} / n_{M}$, each point in Fig. $7 \mathrm{~A}$ is scaled along the horizontal and vertical axes by the corresponding $n_{L}$ and $n_{M}$, respectively. Consequently, the line for the red-green mixtures and the line for the yellow lights become increasingly more parallel to each other when $n_{L} / n_{M}$ deviates more from unity, this in turn increases the overlap between the two noise-thickened lines near their intersection to make $f_{\text {matching-range larger. }}$

The impact of $n_{L} / n_{M}$ on $f_{\text {matching-range }}$ (particulary in normal trichromats) is a prediction that can be tested. However, $f_{\text {matching-range }}$ also changes with the cone sensitivities $\left(\left(r_{L}, r_{M}\right)\right.$, $\left(g_{L}, g_{M}\right)$, and $\left.\left(y_{L}, y_{M}\right)\right)$ which also determine $f_{\text {match }}$. Holding $f_{\text {match }}$ fixed does not nail down $f_{\text {matching-range, since there are }}$ four underlying variables $\left(\lambda_{\max }\right.$ and $O_{c}$ for each of the $\mathrm{L}$ and $\mathrm{M}$ cone types) to determine the cone sensitivities (assuming the anomaloscope and the pre-receptor retinal filters are fixed). Once $n_{L}=n_{M}=0.5$ is fixed, collective variations of each $\lambda_{\max }$ within the range of 3-4 nm and each $O_{C}$ within a factor of 23 can cause $f_{\text {matching-range }}$ to vary within a range of about two fold. Therefore, testing the predicted effect of cone numerosity on $f_{\text {matching-range }}$ should require either a large number of observers to average out the variations caused by the variations of cone sensitivities, or a group of observers whose cone sensitivities (or related specifics) are precisely known.

\section{G. Matching difficulties and smaller matching ranges for strong tetrachromats}

Human tetrachromats are observers who have four functioning types of cones. We consider those tetrachromats who have three functioning types of cones which are, unlike the $S$ cones, sensitive to the wavelengths of the red, green, and yellow lights for the Rayleigh match. For example, a heterozygous female carrier of anomalous trichromacy can possibly be a tetrachromat by having a normal L cone, a normal $\mathrm{M}$ cone, and an abnormal L or abnormal $\mathrm{M}$ cone[19]. We consider the case of strong tetrachromats when post-receptor processing preserves the extra dimension of color information by the additional cone type (as opposed to weak tetrachromats when the extra color dimension is lost post-receptorally)[20]. For these individuals, a match requires that each of the three (rather than two) types of cones is activated equally by the two light fields, one red-green mixture and one yellow light. When there is no noise in cone activations, such a match is not mathematically possible since it would require satisfying simultaneously three equations, one for each cone type, by only adjusting two variables, the mixing fraction $f$ and the yellow-light intensity. Graphically, this impossibility can be understand by generalizing Fig 2A from a two-dimensional space to a three dimensional space, with the three axes representing the respective responses from the three cone types. In this three dimensional space, two lines of the cone responses, one for the red-green mixture and one for the yellow light, are unlikely to intersect each other for the exact match. In practice, when cone activation noise is considered, however, a match may still be possible for such a tetrachromat if the closest distance or gap between the two lines is within the noise range - the $f$ value and the yellow light intensity to achieve this closest distance is then the matching point and the noise amplitude is sufficient to bridge the gap. If the noise is not sufficient to bridge the gap, such a tetrachromat would not be able to find a match.

For tetrachromats who can achieve a match, the matching range is likely smaller than usual because the above mentioned 
gap makes it more difficult for any fraction $f$ to achieve a match even with the cone noise. This smaller matching range can also be understood using the example when the relevant three cone types consist of the normal $\mathrm{L}$ cone, the normal $\mathrm{M}$ cone, and an abnormal L cone. A match involving these three cone types is equivalent to two simultaneous two-cone matches, one involving only the normal $\mathrm{L}$ cone and the normal $\mathrm{M}$ cone, another involving only the abnormal $\mathrm{L}$ cone and the normal $\mathrm{M}$ cone. The matching range for the three-cone match should be the intersection between two matching ranges for the two respective two-cone matches, and therefore should be smaller than usual. For example, if the matching range for the Rayleigh matching involving the normal $\mathrm{L}$ and $\mathrm{M}$ cones is $f_{1} \in(0.48,0.51)$ and that for a match involving the abnormal $\mathrm{L}$ and the normal $\mathrm{M}$ cones is $f_{2} \in(0.50,0.60)$, then the matching range involving all the three cones is $f \in(0.50,0.51)$. If the first two matching ranges do not overlap, then the tetrachromat will not find any fraction $f$ to achieve a satisfactory match.

\section{SUMMARY AND DISCUSSIONS}

We introduce an analytical model of the Rayleigh match, so that the matching fraction $f_{\text {match }}$ is expressed by an explicit mathematical formula (in equation (10)), showing how $f_{\text {match }}$ depends on the three L-M pairs of cone sensitivities $\left(\left(r_{L}, r_{M}\right)\right.$, $\left(g_{L}, g_{M}\right)$, and $\left.\left(y_{L}, y_{M}\right)\right)$ to the three lights (the sensitivities in turn are precisely determined by the $\lambda_{\text {max }}$ and optical densities of photopigments, properties of the pre-receptor retinal filter, and the wavelengths (or spectrum compositions) of the three lights for the anomaloscope) and on the intensities $R$ and $G$ of the red and green lights. In addition, we propose that the noise in the cone responses are responsible for the non-zero matching range, and used the Poisson nature of the noise in the cones to derive another mathematical formula for the matching range (in equation (23) or, as an approximation for normal trichromats, equation (25)). The matching range is shown to be influenced not only by the same retinal and anomaloscope quantities $\left(\left(r_{L}, r_{M}\right),\left(g_{L}, g_{M}\right),\left(y_{L}, y_{M}\right), R\right.$, and $\left.G\right)$ that determined the matching fraction $f_{\text {match }}$, but also by the relative numbers $n_{L}$ and $n_{M}$ of $\mathrm{L}$ and $\mathrm{M}$ cones.

We also introduced a graphical understanding of the Rayleigh match, as shown for example in Figs. 2, 5, 6, and 7. Together, the graphical understanding and the analytical formulae enabled us to understand why dichromats can match at any red-green mixtures, why protanomalous needs more red light and deuteranomalous need more green light for the match, why cone absorption noise makes matching range non-zero, why anomalous trichromats have larger matching ranges than the normal trichromats, and how relative $\mathrm{L}$ and $\mathrm{M}$ cone numerosities influence the matching range.

In addition, our model and understanding provide the following four predictions which can be tested by experimental data.

1. Everything else being equal, matching range in normal trichromats is larger when $\mathrm{L}$ and $\mathrm{M}$ cones have very different relative numerosities. For example, when there are ten times as many cones of one type as that of the other type, the matching range $f_{\text {matching-range }}$ can be about two or three times as large as that when the two types of cones have comparable numerosities.

2. Female normal trichromats who are also carriers of dichromacy (i.e., they have one $\mathrm{X}$-chromosome with genes for the normal cones and another X-chromosome lacking the gene for the L or M cone) are more likely to have larger matching ranges than non-carrier normal trichromats.

3. On average, the matching range in the female deutan carriers is larger than that in the female protan carriers.

4. The matching range in strong tetrachromats is typically smaller than that in the typical normal trichromats, and that the Rayleigh matching is impossible in some strong tetrachromats.

Next, we elaborate and discuss on these predictions.

For prediction (1), since, given numerosities of the cones, $f_{\text {matching-range }}$ can also vary by two to three fold due to variations in $\lambda_{\max }$ and optical densities of the photopigments among the normal trichromats, the predicted effects by the numerosities of the cones is best tested among observers having the same sensitivites of the cones. Alternatively, we can also employ many observers so that variabilities of $f_{\text {matching-range }}$ caused by the variabilities of the cone sensitivites can be averaged out among the observers. We note that, due to variabilities in both the numerosities and sensitivities of the cones among normal trichromats, $f_{\text {matching-range }}$ can vary by up to about 10 fold. A previous work[8] had speculated that this roughly 10 fold change may be caused by the same roughly 10 fold change in the relative numerosities of the $\mathrm{L}$ and $\mathrm{M}$ cones among the normal trichromats[21, 22]. Our analytical modeling however enables us to predict that a 10 fold change in the relative numerosities only causes about $2-3$ fold change in $f_{\text {matching-range }}$.

Prediction (2) is a corollary of prediction (1) since female normal trichromats who are also carriers of dichromacy are more likely to have extreme $n_{L} / n_{M}$ values. Ignoring the $\mathrm{S}$ cones for simplicity, if each photoreceptor randomly expresses one of the $\mathrm{L}$ and $\mathrm{M}$ genes from only one of the X-chromosomes[23], the retina is more likely to have fewer cones of the type whose gene is lacking in the dichromacy X-chromosome. In particular, we can calculate the modified fraction $n_{L}^{\prime}$ of the L cones in these carriers using a simple model as follows. Assume that (1) only one X-chromosome is activated to express a cone for each photoreceptor, and let $p$ be the chance that this X-chromosome is the normal one; and (2), if the normal X-chromosome is activated, an $\mathrm{L}$ or $\mathrm{M}$ cone is expressed with a probability $n_{L}$ or $n_{M}$, respectively, like in a non-carrier normal trichromat, and when the abnormal, deutan or protan, X-chromosome is activated, the gene expressed is always for an L or M cone, respectively. The modified $n_{L}^{\prime}$ is then the overall probability that an $L$ cone gene is expressed. Hence, for the protan carrier, this probability is

$$
n_{L}^{\prime}(\text { protan carrier })=p \cdot n_{L},
$$

since a L cone only occurs when the normal X-chromosome is activated and also an L gene is chosen for expression. Similarly, for the deutan carrier (note that $n_{L}+n_{M}=1$ ),

$$
\begin{aligned}
n_{L}^{\prime}(\text { deutan carrier }) & =p \cdot n_{L}+(1-p) \\
& =1-p \cdot n_{M} .
\end{aligned}
$$

In equation (31), the first term $p \cdot n_{L}$ after the equal sign is the same as before and the second term, $(1-p)$, is the probability that the abnormal X-chromosome (which can only express an $\mathrm{L}$ cone) is activated. Hence, relative to non-carrier normal trichromats, the fraction of $\mathrm{L}$ cones is, on average, reduced in protan carriers and increased for deutan carriers. In either case, the distribution of $\mathrm{L} / \mathrm{M}$ ratio deviates from that of the non-carrier normal trichromats. In particular, a small $p$ value can lead to very 
small $n_{L}^{\prime}$ for protan carriers[24] and very large $n_{L}^{\prime}$ for deutan carriers. The chance for $p$ much smaller than $1 / 2$ is quite substantial, since the activated X-chromosome in each cell is inherited from a very small pool of (around 7-8) precursor cells early in female embryological development[25]. Since our model predicts that extreme L cone fractions lead to larger $f_{\text {matching-range }}$ values, female carriers of dichromacy should have on average a larger $f_{\text {matching-range }}$ value. This may explain the observation that such carriers have on average an $f_{\text {matching-range that are }}$ about 2-3 times of that for non-carrier normal trichromats[19], though this difference in $f_{\text {matching-range }}$ has not been observed in other studies[26]. Regardless, our analysis above also leads to prediction (3) that a larger $f_{\text {matching-range }}$ is more likely to occur in a deutan than a protan carrier. This is because typically $n_{L}>n_{M}$ in normal non-carriers, hence, given each $p$ value,

$$
n_{L}^{\prime}(\text { protan carrier })=p n_{L}>1-n_{L}^{\prime}(\text { deutan carrier })=p n_{M} \text {. }
$$

This means, the distance of $n_{L}^{\prime}$ (protan carrier) from the extreme 0 is larger than the distance of $n_{L}^{\prime}$ (deutan carrier) from the extreme 1 , i.e., extreme $L$ cone fractions are more likely in deutan than protan carriers. Predictions (2) and (3) regarding the $f_{\text {matching-range }}$ in female carriers must be tested using sufficiently large (larger than those from the observations so far $[19,26])$ pools of female carriers for whom the spectral sensitivity of their underlying pigments is known and for whom the relative ratio of L:M cones is known. New imaging techniques may allow for L:M ratio to be estimated more expeditiously in these subjects[27].

Now we come to prediction (4) on the strong tetrachromats. Both analytically and intuitively, when the fourth, abnormal, cone in the tetrachromats is more like one of the normal cones in terms of its sensitivities to the three lights, it is more likely for such a tetrachromat to find a match, since such a tetrachromat is more like a normal trichromat. We should also consider the possibility of (female) tetrachromats who carry two different versions of abnormal $\mathrm{L}$ or $\mathrm{M}$ cones. For example, consider an individual with normal $\mathrm{L}$ cones but two different versions of the abnormal $\mathrm{M}$ cones. This individual would appear like a deuteranomalous who requires more green light than normal to match; however, she would be likely to have a smaller matching range than that of a typical (trichromatic) deuteranomalous.

Only females can be (strong) tetrachromats or normal trichromats who are also carriers of dichromacy. In the former case, our model predicts that their matching range should be smaller; in the latter case, their matching range is predicted to be likely larger. Hence, between males and females who are not color vision defficient, i.e., not dichromats or anomalous trichromats, there may or may not be a significant difference between the two genders in terms of the average matching range. Interestingly, Rodriguez-Carmona et al[28] observed a larger matching range in a sample of females believed to be absent of any carriers of color vision deficiency. However no genetic testing was performed in that study, and the statistical method of "removing" carriers from their sample is not ideal. To obtain clean data, it is best to test each prediction using only the corresponding group of females without contamination from the other group. Furthermore, it has been noted that most heterozygous females who are carriers of anomalous trichromacy are not strong tetrachromats who have their four dimensional color space preserved at the post-receptor level[19, 20, 29]. It is thus important to exclude the weak tetrachromatic subjects when testing the prediction about the tetrachromats, since these weak tetrachromatics could have complex color matching behavior caused by their likely extra color dimension at the receptor, but not post-receptor, level[30]. Our formulation of the Rayleigh matching applies only when the dimensions of color information at the receptor level are preserved post-receptorally. Tests of various predictions of our modeling will help shed light on the mechanisms by which color signals are processed by the human visual system.

\section{FUNDING INFORMATION}

Funding. The research reported in this publication was supported by (to L. Zhaoping) the Gatsby Charitable Foundation and (to J. Carroll) by the National Eye Institute of the National Institutes of Health under award numbers P30EY001931 and R01EY017607. The content is solely the responsibility of the authors and does not necessarily represent the official views of the funders.

\section{ACKNOWLEDGMENTS}

We are grateful for the helpful comments by John L. Barbur.

\section{REFERENCES}

1. L. Rayleigh, "Experiments on colour," Nature 25, 64-66 (1881).

2. W. A. Nagel, "I. zwei apparate für die augenärztliche funktionsprüfung," Ophthalmologica 17, 201-222 (1907).

3. L. T. Sharpe, A. Stockman, H. Jägle, and J. Nathans, "Opsin genes, cone photopigments, color vision, and color blindness," in "Color vision: from genes to perception,", K. R. Gegenfurtner and L. T. Sharpe, eds. (Cambridge University Press, New York, 1999), pp. 3-52.

4. J. Neitz and G. H. Jacobs, "Polymorphism of the long-wavelength cone in normal human colour vision," Nature 323, 623-625 (1986).

5. J. Pokorny, V. C. Smith, and I. Katz, "Derivation of the photopigment absorption spectra in anomalous trichromats," Journal of Optical Society of America 63, 232-237 (1973).

6. J. C. He and S. K. Shevell, "Variation in color matching and discrimination among deuteranomalous trichromats: Theoretical implications of small differences in photopigments," Vision Research 35, 2579-2588 (1995).

7. P. Thomas and J. Mollon, "Modelling the Rayleigh match," Visual Neuroscience 21, 477-482 (2004).

8. J. Barbur, M. Rodriguez-Carmona, J. Harlow, K. Mancuso, J. Neitz, and M. Neitz, "A study of unusual Rayleigh matches in deutan deficiency," Visual Neuroscience 25, 507-516 (2008).

9. T. Lamb, "Photoreceptor spectral sensitivities: common shape in the long-wavelength region," Vision Research 35, 3083-3091 (1995).

10. A. Stockman and L. T. Sharpe, "The spectral sensitivities of the middle-and long-wavelength-sensitive cones derived from measurements in observers of known genotype," Vision Research 40, 17111737 (2000).

11. P. Lillywhite and S. Laughlin, "Transducer noise in a photoreceptor," Nature 277, 569-572 (1979).

12. H. Barlow, "Retinal and central factors in human vision limited by noise," in "Vertebrate Photoreception," , H. Barlow and P. Fatt, eds. (Academic Press, London, 1977), pp. 337-358.

13. L. Zhaoping, W. Geisler, and K. May, "Human wavelength discrimination of monochromatic light explained by optimal wavelength decoding of light of unknown intensity," PLoS One 6, e19248 (2011).

14. L. Zhaoping, Understanding Vision: theory, models, and data (Oxford University Press, 2014).

15. J. Birch, "Classification of anomalous trichromatism with the nagel anomaloscope," in "Colour Vision Deficiencies XI, Volume 56 of the series Documenta Ophthalmologica Proceedings Series," (Springer, 1993), pp. 19-24. 
16. M. Neitz, J. Carroll, A. Renner, H. Knau, J. S. Werner, and J. Neitz, "Variety of genotypes in males diagnosed as dichromatic on a conventional clinical anomaloscope," Visual neuroscience 21, 205-216 (2004).

17. J. Barbur and M. Rodriguez-Carmona, "Variability in normal and defective colour vision: Consequences for occupational environments," in "Colour design," , J. Best, ed. (Cambridge:Woodhead Publishing Limited, 2012), pp. 24-82.

18. R. C. Baraas, D. H. Foster, K. Amano, and S. M. Nascimento, "Color constancy of red-green dichromats and anomalous trichromats," Investigative ophthalmology \& visual science 51, 2286-2293 (2010).

19. G. Jordan and J. Mollon, "A study of women heterozygous for colour deficiencies," Vision Research 33, 1495-1508 (1993).

20. A. L. Nagy, D. I. MacLeod, N. E. Heyneman, and A. Eisner, "Four cone pigments in women heterozygous for color deficiency," Journal of the Optical Society of America 71, 719-722 (1981).

21. G. H. Jacobs and J. Neitz, "Electrophysiological estimates of individual variation in the $\mathrm{I} / \mathrm{m}$ cone ratio," in "Colour vision deficiencies $\mathrm{XI}$," (Springer, 1993), pp. 107-112.

22. J. Carroll, J. Neitz, and M. Neitz, "Estimates of I: M cone ratio from ERG flicker photometry and genetics," Journal of Vision 2, 531-542 (2002).

23. M. F. Lyon, "Gene action in the X-chromosome of the mouse (Mus musculus L.)," Nature pp. 372-373 (1961).

24. E. Miyahara, J. Pokorny, V. C. Smith, R. Baron, and E. Baron, "Color vision in two observers with highly biased Iws/mws cone ratios," Vision Research 38, 601-612 (1998).

25. G. H. Jacobs and G. A. Williams, " $L$ and $m$ cone proportions in polymorphic new world monkeys," Visual Neuroscience 23, 365-370 (2006).

26. E. Konstantakopoulou, M. Rodriguez-Carmona, and J. L. Barbur, "Processing of color signals in female carriers of color vision deficiency," Journal of vision 12, 11 (2012)

27. R. Sabesan, H. Hofer, and A. Roorda, "Characterizing the human cone photoreceptor mosaic via dynamic photopigment densitometry," PloS one 10, e0144891 (2015).

28. M. Rodriguez-Carmona, L. Sharpe, J. Harlow, and J. Barbur, "Sexrelated differences in chromatic sensitivity," Visual neuroscience $\mathbf{2 5}$, 433-440 (2008).

29. G. Jordan, S. S. Deeb, J. M. Bosten, and J. Mollon, "The dimensionality of color vision in carriers of anomalous trichromacy," Journal of Vision 10, 12 (2010).

30. Y. Sun and S. K. Shevell, "Rayleigh matches in carriers of inherited color vision defects: The contribution from the third I/m photopigment," Visual Neuroscience 25, 455-462 (2008). 